\title{
Conceptual confusion and potential advances in athlete burnout research
}

Henrik Gustafsson, Erik Lundkvist, Leslie Podlog and Carolina Lundqvist

The self-archived postprint version of this journal article is available at Linköping University Institutional Repository (DiVA):

http:/ / urn.kb.se/ resolve?urn=urn:nbn:se:liu:diva-153208

N.B.: When citing this work, cite the original publication.

Gustafsson, H., Lundkvist, E., Podlog, L., Lundqvist, C., (2016), Conceptual confusion and potential advances in athlete burnout research, Perceptual and Motor Skills, 123(3), 784-791.

https:// doi.org/ 10.1177/ 0031512516665900

Original publication available at:

https:/ / doi.org/ 10.1177/ 0031512516665900

Copyright: Ammons Scientific

http:// www.amsci.com/ 
Conceptual Confusion and Potential Advances in Athlete Burnout Research

\author{
Henrik Gustafsson \\ Karlstad University \\ Erik Lundkvist \\ St Andrews University \\ Umeå University \\ Leslie Podlog \\ University of Utah \\ Carolina Lundqvist \\ Karlstad University
}

Correspondence concerning this article should be addressed to Henrik Gustafsson, Faculty of Health, Science and Technology, Karlstad University, 651 88, Karlstad, Sweden. Email: henrik.gustafsson@kau.se. Phone: +46 547001698 


\section{Conceptual Confusion and Potential Advances in Athlete Burnout Research}

More than 30 years of research on athlete burnout has yielded important insights and questions regarding the onset, nature, and consequences of this detrimental syndrome. Not surprisingly, burnout is considered an important matter, both from a research and practical standpoint (cf. Gustafsson, Hancock, \& Coté, 2014). We have been invited to comment on the work of Ryu, Ali, Kim, Choi, and Radlo (2015), who examined the impact of burnout on cognitive performance among athletes.

\section{Novel Advances by Ryu and Colleagues}

A noticeable gap in previous sport burnout research is the failure to determine whether athletes with or without burnout can be distinguished based on psychophysiological markers. By taking EEG’s of athletes’ brains, Ryu et al. (2015) were able to demonstrate variable brain wave patterns among athletes suggested to be burned out or not. This novel finding highlights the fact that psychophysiological differences may be apparent among athletes with or without burnout. A second important contribution of Ryu et al.’s (2015) work was the demonstration that athletes with symptoms suggestive of burnout performed less accurately on the Stroop Color-Word Test. This finding suggests that cognitive deficits may be an outcome of burnout. Despite the important and novel insights emerging from Ryu et al.’s (2015) investigation, we outline a number of concerns regarding the researchers' interpretation of their findings. In particular, issues regarding Ryu et al.'s conceptualization of burnout and their selection of measurement inventories are described. As we believe these two issues extend beyond the work of Ryu et al., we use the current paper as an opportunity to 
outline conceptual and measurement concerns which we believe are germane to sport burnout research more broadly.

\section{What is burnout?}

Although substantial burnout research has been undertaken across various contexts (occupational settings, nursing, and sport), there remains a lack of consensus regarding the definition of the construct (cf., Kristensen, Borritz, Villadsen, \& Christensen, 2005; Lundkvist, Gustafsson, \& Davis, 2015; Shirom, 2005). Many researchers have adopted the three dimensional definition of Maslach and Jackson (1986) in which burnout consists of emotional exhaustion, depersonalization, and reduced personal accomplishment. We believe this definition of burnout is problematic for several reasons. First, a reduced sense of personal accomplishment has consistently been shown to have lower correlations with the other two dimensions and seems to be differently related to other organizational aspects such as job satisfaction and commitment than exhaustion and depersonalization (Halbesleben \& Demerouti, 2005; Lee \& Ashforth, 1996). Second, theoretical questions concerning the nature of the relationship between emotional exhaustion, depersonalization/cynicism, and a reduced sense of accomplishment have never been answered (Shirom \& Melamed, 2006). Third, Maslach's burnout definition is based neither on clinical observations nor on theory. Instead, it has been inductively developed using exploratory factor analysis (Lee \& Ashforth, 1990; Shirom \& Melamed, 2006). Forth, burnout dimensions have substantial conceptual overlap with other constructs used in psychological research. For example, a reduced sense of personal accomplishment includes items that stem from several different constructs such as self-efficacy, productivity, personal competence, and personal 
productivity. In addition, questions regarding how and why emotional exhaustion, depersonalization/cynicism, and a reduced sense of accomplishment can be condensed into a single dimension have not, as yet, been theoretically addressed (Shirom \& Melamed, 2006). Therefore, some researchers have claimed that burnout could be defined based on the core dimension of exhaustion (e.g., Kristiansen et al., 2005; Shirom, 2005). Shiroms definition of burnout however, also showed a problematic connection with depression (Bianchi \& Schonfeld, 2016). Thus, the definition of burnout is an important issue, both in occupational as well as sport settings. This conceptual confusion is of relevance when evaluating Ryu et al.’s (2015) findings.

Another problem related to the interpretation of Ryu et al.'s results - an issue which we believe relates to sport burnout research more broadly - is the lack of theory in conceptualizing the nature of the construct (Schaufeli \& Buunk, 2003; Shirom \& Melamed, 2006). For example, the contention that exhaustion leads to depersonalization, and subsequent perceptions of reduced accomplishment has recieved mixed results in the few studies testing this assumption. Some researchers have found the opposite relationships, in which a reduced sense of personal accomplishment is the initial symptom that leads to depersonalization and eventually to emotional exhaustion (Golembiewski, Munzenrider, \& Stevenson, 1986). Conversely, Lee and Ashforth (1993) demonstrated that emotional exhaustion was the first symptom in the burnout sequale and that the temporal progressions to depersonalization and a lack of personal accomplishment were independent of one another. According to Taris, Le Blanc, Schaufeli, and Schreurs (2005), a key problem with the aforementioned studies examining temporal relations is that scholars haveonly tested their own temporal model. 
When testing all potential models, Taris et al. (2005) found that temporal relations appeared to exist in all of the proposed directions. This finding led Taris and colleagues to the conclusion that sequential relations between the burnout dimensions differ and that focusing only on one proposed theoretical model may be detrimental in furthering burnout research. It is also worth mentioning that beta weights in the study by Taris et al. (2005) were very low and the practical significance of those relations can be questioned. Finally, it should be noted that all studies mentioned above used healthy samples and that it would be informative to test potential relationships among constructs on persons that are in the process of becoming burned out. From a pragmatic standpoint, implementing such studies would be challenging; however, adopting mixture models that enable researchers to separate individuals that seem to have rising scores of burnout would be beneficial (c.f., Jung \& Wickrama, 2008).

\section{Issues surrounding measurement of burnout and exhaustion}

Development of the Athlete Burnout Questionnaire (ABQ; Radeke \& Smith, 2001) has helped advance research on athlete burnout and has been the most commonly used measure (Gustafsson et al., 2014; Raedeke \& Smith, 2009). ABQ is based on the original version of Maslach’s Burnout Inventory (MBI; Maslach \& Jackson, 1986). Psychometric comparisons between the ABQ and MBI in athletic populations have been undertaken (Cresswell \& Eklund, 2006; Raedeke, Arce, Seoane \& De Francisco, 2013). Unfortunately, statistical shortcomings in Cresswell and Eklund (2006) and Raedeke et al.’s (2013) psychometric evaluations make comparisons between the two measures hard to evaluate. Neither of the studies reported factor loadings on general factors in their models, making claims of convergent and discriminant validity difficult to assess. 
Furthermore, according to Eid, Lischetzke, Nussbeck, and Trierweiler (2003), in order to analyze both discriminant and convergent validity, the tested model should be created in a manner that covers how much items that belong to a certain subscale load on a general factor - in this case, the general factors of exhaustion, cynicism/devaluation, and a reduced sense of accomplishment. Unfortunately, Cresswell and Eklund (2006) and Raedeke et al (2013) base their claims of convergent validity on correlations between subscales rather than factor loadings on method specific and general factors, as suggested by Eid et al. (2003). Ryu et al. (2015) used an adapted version of the MBI, the Sport Adaptation of the Maslach Burnout Inventory (SAMBI; Fender, 1988; Song, 1999). The SAMBI has been used in two unpublished doctoral dissertations, but no published studies has provided indication of its psychometric properties. These issues make the choice of SAMBI questionable.

We also take issue with Ryu et al.'s (2015) decision to combine the three subscales into a single burnout score. According to the MBI manual, the three subscales should be measured individually (Maslach Jackson, \& Leiter, 1996). Unfortunately, no indication regarding why the subscales were merged is provided. Had Ryu et al. (2015) analyzed the MBI subscales separately, interpretation of the results would have been made easier, as there is evidence that the exhaustion subscale of MBI can discriminate between workers who are - or are not - on sick leave for stress-related issues (Schaufeli, Bakker, Hoogduin, \& Kladler, 2001). Even if Ryu et al. (2015) had classified athletes as "burned-out" or not based on individual subscale scores, their classification still would not be valid since no clinical cut-off levels from the MBI (or ABQ for that matter) are available. Cut-offs from the MBI manual is based on three equally large groups where the 
.33 and .66 percentiles are used to separate the three groups (Maslach, Jackson \& Leiter, 1996). Being "high" on burnout therefore does not indicate anything more than the fact that the person has a level of burnout that is higher than two thirds of the general American population. There is a need therefore to establish potential cut-offs in an athlete population.

The lack of psychometric evidence for the MBI and ABQ makes the choice of an appropriate burnout measure problematic. We should be the first to admit, that we too have struggled to determine which burnout measure is most optimal, and have typically adopted the ABQ. So, what measure should Ryu and colleagues have used? One option is to use the exhaustion subscale of the $\mathrm{ABQ}$ only, as there appears to be some consensus among researchers that exhaustion is likely the core dimension of burnout (c.f., Gustafsson, Kenttä, \& Hassmén, 2011; Maslach, Schaufeli, \& Leiter, 2001; Shirom, 2005), and as been mentioned, shown to descriminate workers on stress-related sick leave. Another alternative is to use a more generic and clinically validated measure. For example, with the Shirom-Melamed Burnout Questionnaire (Lerman et al., 1999), the results could have been interpreted in relation to clinical cut-offs, thereby making the meaningfulness of the results easier to interpret. A third potential measure that Ryu and colleagues could have selected is the Karolinska Exhaustion Disorder Scale (KEDS; Beser, Sorjonen, Wahlberg, Peterson, Nygren \& Åsberg, 2014). The scale is based on an exhaustion disorder diagnosis which is clinically valid and has a broad empirical and theoretical base. It has recently been argued that exhaustion disorder is the clinical manifestation of burnout (Grossi, Perski, Osika, \& Savic, 2015). 
In advocating the above burnout measures, we acknowledge that they have yet to be validated in an athletic context and thefore require further psychometric testing. Nonetheless, we would still advocate using scales where clinical cut offs are available, rather than instruments validated with athletes, since it remains uncertain whether athletes have different burnout perceptions (i.e., scores) than members of the general population - of which athletes could of course be included. Given an absence of information pointing to any such differences,we suggest that adopting an instrument with clinical cut offs may be more effective in identifying the point at which burnout levels become problematic for healthy psychosocial functioning, well-being, and performance. Thus being able to measure problematic levels of burnout, we suggest adopting clinical validated measures.

\section{Future research directions}

The finding that athletes with higher scores on SAMBI suffered impaired performance on the Stroop Color-Word compared to their low burnout counterparts is interesting. Recent research has also pointed to the fact that cognitive performance may be compromised among those suffering from burnout in comparision to healthy controls. For example, Sandström et al. (2011) found that patients suffereing from stress-releated exhaustion performed significantly poorer on measures of attention and response control, as well as visuo-spatial memory ability. This research highlights the fact that individuals suffering health concerns - such as athletes’ experiencing burnout - may also struggle with cognitive tasks and functioning that impair their decision making ability and ultimate sport performance. Further research is needed to test this effects in sport but the findings from Ryu and colleagues can be an interesting start for research in sport. 
As highlighted above, further work is needed to development conceptually sound burnout measures in a sport context. Although, the ABQ (Raedeke \& Smith, 2001), has done much to advance the area of athlete burnout research, there is a need for further development. We agree, as stated in the ABQ-manual (Raedeke \& Smith, 2009), that a valuable direction for future research would be to test ABQ-scores in association to a theoretical or clinical framework, in order to delinate what constitutes high burnout. In the meantime, clinically validated measures can be an alternative to discriminate high burnout from low burnout.

\section{References}

Besèr, A., Sorjonen, K., Wahlberg, K., Peterson, U., Nygren, Å., \& Åsberg, M. (2014). Construction and evaluation of a self-rating scale for stress-induced Exhaustion Disorder, the Karolinska Exhaustion Disorder Scale. Scandinavian Journal of psychology, 55(1), 72-82.

Bianchi, R., \& Schonfeld, I. S. (2016). Burnout is associated with a depressive cognitive style. Personality and Individual Differences, 1-5. http://doi.org/10.1016/j.paid.2016.01.008

Cresswell, S. L., \& Eklund, R. C. (2006). The convergent and discriminant validity of burnout measures in sport: A multi-trait/multi-method analysis. Journal of Sports Sciences, 24(2), 209-220.

Eid, M., Lischetzke, T., Nussbeck, F. W., \& Trierweiler, L. I. (2003). Separating trait effects from trait-specific method effects in multitrait-multimethod models: A multiple-indicator CT-C(M-1) model. Psychological Methods, 8(1), 38-60. doi.org/10.1037/1082-989X.8.1.38

Glass, D. C., \& McKnight, J. D. (1996). Perceived control, depressive symptomatology, and professional burnout: A review of the evidence. Psychology and Health, 11(1), 23-48.

Golembiewski, R. T., Munzenrider, R. F., \& Stevenson, J. G. (1986). Stress and organizations: Toward a phase model of burnout. New York: Praeger.

Grossi, G., Perski, A., Osika, W., \& Savic, I. (2015). Stress-related exhaustion disorderclinical manifestation of burnout? A review of assessment methods, sleep 
impairments, cognitive disturbances, and neuro-biological and physiological changes in clinical burnout. Scandinavian Journal of Psychology, 56(6), 626-636.

Gustafsson, H., Hancock, D., \& Coté, J. (2014). Describing citation structures in sport burnout literature: A citation network analysis. Psychology of Sport \& Exercise, 15, 620-625.

Gustafsson, H., Kenttä, G., \& Hassmén, P. (2011). Athlete burnout: An integrated model and future Directions. International Review in Sport and Exercise Psychology, 4, 324.

Jung, T., \& Wickrama, K. a. S. (2008). An Introduction to Latent Class Growth Analysis and Growth Mixture Modeling. Social and Personality Psychology Compass, 2(1), 302-317. doi.org/10.1111/j.1751-9004.2007.00054.x

Kristensen, T. S., Borritz, M., Villadsen, E., \& Christensen, K. B. (2005). The Copenhagen Burnout Inventory: A new tool for the assessment of burnout. Work \& Stress, 19(3), 192-207. http://doi.org/10.1080/02678370500297720

Lee, R. T., \& Ashforth, B. E. (1990). On the meaning of Maslach's three dimensions of burnout. Journal of Applied Psychology, 75(6), 743-747. doi.org/10.1037/00219010.75.6.743

Lee, R. T., \& Ashforth, B. E. (1993). A longitudinal study of burnout among supervisors and managers: comparisons between the Leiter and Maslach (1988) and Golembiewski et al. (1986) models. Organizational Behavior and Human Decision Processes, 54, 369-398.

Lee, R. T., \& Ashforth, B. E. (1996). A meta-analytic examination of the correlates of the three dimensions of job burnout. The Journal of Applied Psychology, 81(2), 123-33.

Lundkvist, E., Gustafsson, H., \& Davis, P. A. (2015). What is missing and why it is missing from coach burnout research. In P. A. Davis (Ed.), The psychology of effective coaching and management (1st ed., pp. 407-427). New York, NY: Nova Science Publishers.

Maslach, C., Jackson, S., \& Leiter, M. P. (1996). Maslach Burnout Inventory: Manual (3:rd ed.). Paulo Alto: Consulting Psychologist Press.

Raedeke, T. D., Arce, C., De Francisco, C., Seoane, G., \& Ferraces, M. J. (2013). The construct validity of the Spanish version of the ABQ using a multi-trait/multimethod approach. Anales de Psicología, 29(3), 693-700.

Raedeke, T. D., \& Smith, A. L. (2001). Development and preliminary validation of an athlete burnout measure. Journal of Sport and Exercise Psychology, 23, 281-306. 
Raedeke, T. D., \& Smith, A. L. (2009). The athlete burnout questionnaire manual. Fitness Information Technology.

Sandström, A., Peterson, J., Sandström, E., Lundberg, M., Nyström, I. L. R., Nyberg, L., \& Olsson, T. (2011). Cognitive deficits in relation to personality type and hypothalamic-pituitary-adrenal (HPA) axis dysfunction in women with stress-related exhaustion. Scandinavian Journal of Psychology, 52(1), 71-82.

Shirom, A. (2005). Reflections on the study of burnout The views expressed in Work \& Stress commentaries are those of the author(s), and do not necessarily represent those of any other person or organization, or of the journal. Work \& Stress, 19(3), 263-270. http://doi.org/10.1080/02678370500376649

Shirom, A., \& Melamed, S. (2006). A comparison of the construct validity of two burnout measures in two groups of professionals. International Journal of Stress Management, 13(2), 176-200. doi.org/10.1037/1072-5245.13.2.176

Taris, T. W., Le Blanc, P. M., Schaufeli, W. B., \& Schreurs, P. J. G. (2005). Are there causal relationships between the dimensions of the Maslach Burnout Inventory? A review and two longitudinal tests. Work \& Stress, 19(3), 238-255. doi.org/10.1080/02678370500270453 